\title{
Erratum to: Endogenous soluble receptor of advanced glycation end-products (esRAGE) is negatively associated with vascular calcification in non-diabetic hemodialysis patients
}

\author{
Mohamed M. NasrAllah • Amal R. El-Shehaby • \\ Noha A. Osman • Mona M. Salem • Amr Nassef • \\ Usama A. A. Sharaf El Din
}

Published online: 18 November 2011

(C) Springer Science+Business Media, B.V. 2011

\section{Erratum to: Int Urol Nephrol DOI 10.1007/s11255-011-0007-x}

Unfortunately, the original version of this article published with mistakes. In the Tables 2, 3 and 4 the values of the $95 \%$ Confidence Intervals for esRAGE are incorrect. The correct versions of the tables are given below.

The online version of the original article can be found under doi:10.1007/s11255-011-0007-x.

M. M. NasrAllah $(\bowtie) \cdot$ N. A. Osman · U. A. A. S. El Din Department of Nephrology, Kasr El-Aini School of Medicine, Cairo University, Cairo, Egypt e-mail: mmnasralla@yahoo.com

\section{A. R. El-Shehaby}

Department of Medical Biochemistry, Kasr El-Aini School of Medicine, Cairo University, Cairo, Egypt

M. M. Salem

Department of Endocrinology, Kasr El-Aini School of Medicine, Cairo University, Cairo, Egypt

\section{A. Nassef \\ Department of Radiology, Kasr El-Aini School of Medicine, Cairo University, Cairo, Egypt}

\section{U. A. A. S. El Din}

Department of Nephrology, Kasr El-Aini School of Medicine, Cairo University and International Kidney Centre, Cairo, Egypt
Table 2 Multiple regression analysis of factors associated with aortic calcification index (model excluding hsCRP), $R^{2}$ 0.55

\begin{tabular}{lrcl}
\hline & \multicolumn{1}{l}{$\beta$} & \multicolumn{1}{l}{ P } & 95\% CI \\
\hline esRAGE & -0.49 & $<0.0001$ & -0.086 to -0.034 \\
Systolic BP & 0.43 & $<0.0001$ & $0.1-0.4$ \\
Age & 0.29 & 0.014 & $0.07-0.52$ \\
Vintage & 0.23 & 0.04 & $0.002-0.14$ \\
Phosphate & 0.18 & 0.13 & $-0.24-2$ \\
Parathormone & -0.06 & 0.6 & $-0.01-0.005$ \\
Triglycerides & -0.07 & 0.5 & $-0.05-0.03$ \\
Cholesterol & -0.02 & 0.8 & $-0.06-0.05$ \\
Calcium & 0.036 & 0.72 & $-1.9-2.8$ \\
\hline
\end{tabular}

Table 3 Multiple regression analysis of factors associated with aortic calcification index (model including hsCRP), $R^{2} 0.69$

\begin{tabular}{lccl}
\hline & $\beta$ & $P$ & $95 \%$ CI \\
\hline hsCRP & 0.48 & $<0.0001$ & $0.7-1.7$ \\
Systolic BP & 0.39 & $<0.0001$ & $0.13-0.34$ \\
esRAGE & -0.24 & 0.02 & -0.05 to -0.005 \\
Vintage & 0.3 & 0.02 & $0.01-0.13$ \\
Phosphate & 0.15 & 0.14 & $-0.2-1.7$ \\
Age & 0.13 & 0.17 & $-0.8-3.5$ \\
Parathormone & -0.113 & 0.22 & $-0.01-0.003$ \\
Triglycerides & -0.1 & 0.24 & $-0.05-0.04$ \\
Cholesterol & -0.04 & 0.7 & $-0.06-0.04$ \\
Calcium & 0.1 & 0.2 & -0.8 to -3.3 \\
\hline
\end{tabular}


Table 4 Stepwise multiple regression analysis of factors associated with aortic calcification index

\begin{tabular}{lcll}
\hline & $\beta$ & $P$ & $95 \%$ CI \\
\hline$R^{2}=0.65$ & & & \\
hsCRP & 0.48 & $<0.0001$ & $0.76-1.7$ \\
Systolic BP & 0.4 & $<0.0001$ & $0.1-0.3$ \\
esRAGE & -0.28 & 0.002 & -0.056 to -0.013 \\
Vintage & 0.16 & 0.05 & $0.001-0.098$ \\
\hline
\end{tabular}

